\title{
Pion photoproduction in the delta resonance region
}

\author{
R.A. Arndt, I.I. Strakovsky and R.L. Workman \\ Department of Physics, Virginia Polytechnic Institute and State University, Blacksburg, VA 24061
}

(September 7, 2018)

\begin{abstract}
We have updated our multipole analyses to incorporate new data from the low-energy and delta resonance regions. We note a slight decrease in our estimate of the delta photo-decay amplitudes. This agrees with results found in Compton scattering. Our values are also in good agreement with a previous RPI determination. We have reexamined our determinations of the E2/M1 ratio at the resonance energy and at the pole. We find an E2/M1 ratio (at the pole) which is in good agreement with recent the Mainz value.
\end{abstract}

PACS numbers: 11.80.Et, 13.60.Rj, 25.20.Lj

Typeset using REVTEX 


\section{INTRODUCTION}

Recent interest in the pion photoproduction reaction has been focused on the threshold and delta resonance regions. Most studies have been motivated by a desire to check the predictions of chiral perturbation theory and to pin down the photo-decay amplitudes of the $\Delta(1232)$. With the flood of new and precise data in this region, we have reexamined the $\Delta(1232)$ photo-decay amplitudes coming from our analyses of the pion photoproduction data base. Given the recent interest in a more precise determination of the E2/M1 ratio, we have extracted this quantity as well.

Our method of analysis is the same as was used to generate the results of Ref. [1], with one modification. The $\pi \mathrm{N}$ interaction is currently being determined by the pion C.M. momentum rather than the C.M. energy. This gives a proper threshold behavior for all charge-channel multipoles but shifts the on-shell resonance points (in $\mathrm{W}_{C . M}$.) slightly according to the specific charge channel being considered. The pole position is not altered.

\section{THE DELTA PHOTO-DECAY AMPLITUDES}

Most important to the present analysis were the recent total cross section measurements of Ref. [2]. Our previous analysis [1] predicted total $\pi^{0} p$ and $\pi^{+} n$ total cross sections systematically above these new data. This was also suggested in an analysis of Compton scattering data [3]. In that work, a $2.8 \pm 0.93 \%$ reduction in our $M_{1+}^{3 / 2}$ amplitude was found to be necessary.

Our present findings complement, and now agree with, the value found in Ref. [3]. For the helicity $1 / 2$ and $3 / 2$ photo-decay amplitudes, we find values about $4 \%$ lower than our previous determinations. These are compared to values from the Glasgow [4 and RPI [5] groups in Table I. Though the RPI values have larger errors, the central values from the

VPI and RPI [5] determinations are in good agreement. The ranges for $A_{1 / 2}$ and $A_{3 / 2}$ were chosen to cover results found in fits to our global solution to $2 \mathrm{GeV}$ (SP97), a more restricted 
analysis to $500 \mathrm{MeV}$ (W500), and fits to our single-energy solutions (SES) [6].

\section{THE E2/M1 RATIO}

There are a number of ways to define the E2/M1 ratio. In the following, we will restrict ourselves to determinations which can be made directly from the multipole amplitudes. The

resonance value which we quote is determined by evaluating the ratio $\operatorname{Im} E_{1+}^{3 / 2} / \operatorname{Im} M_{1+}^{3 / 2}$ at the resonance energy. Both the RPI [5] and VPI [7] groups have found ratios near $-1.5 \%$ in the past. However, while the PDG [8] quotes these values, a number of recent determinations have found larger negative values.

Khandaker and Sandorfi [9] have suggested that a higher value is required to fit the new LEGS beam-asymmetry $(\Sigma)$ data [10]. This claim is supported in a recent paper from the Mainz group [11]. A value consistent with the finding of Ref. 9] was found in an analysis of the new Mainz [11] cross section and $\Sigma$ data. A second Mainz analysis [12] used dispersion relations in analyzing data over the delta resonance region. In this work, the speed-plot method was used to determine an E2/M1 ratio at the pole.

In Table II, we compare our updated values for the E2/M1 ratio at the resonance and the pole with both recent and older determinations. We find good agreement with the Mainz pole value, supporting the view that this quantity is relatively model-independent. Adding the new Mainz data did not increase the E2/M1 ratio as much as was found in Ref. [11]. We attribute this to differing assumptions and data bases used in the analyses.

The SES results favor a slightly larger E2/M1 ratio than was found in our energydependent fits. Depending on how the data are binned (we compared results using $10 \mathrm{MeV}$ and $25 \mathrm{MeV}$ bins), the SES value ranges between approximately $-1.5 \%$ and $-1.9 \%$. This value is closer to the one found in Ref. [11]. However, the low-energy analysis (W500), which results in a very different E2/M1 ratio $(-1.2 \%)$, fits the Mainz cross section and $\Sigma$ data [11] quite well. (The overall $\chi^{2} /$ data is $193 / 182$.) This variability in the E2/M1 ratio is greatly reduced in the ratio of pole residues. 
While we found good agreement for the pole positions and moduli of the residues using the speed plot method, we also determined the pole value for E2/M1 using a different method. Writing our amplitudes in the form

$$
M=A_{B}\left(1+i T_{\pi N}\right)+A_{R} T_{\pi N}
$$

where $T_{\pi N}$ is the associated $\pi N$ T-matrix, we fit $T_{\pi N}$ using analytic forms and extrapolated to the pole. ( $A_{B}$ and $A_{R}$ are analytic functions determined in the energy-dependent analysis.) The agreement between these different methods gives further confidence in the pole value. A comparison with the Mainz value [12] is given in Table II. The modulus of this ratio varied by only about $10 \%$ in our fits to SP97, W500, and the SES.

\section{SUMMARY AND CONCLUSIONS}

Our updated $\Delta(1232)$ photo-decay amplitudes now agree with both the results from Compton scattering and an earlier RPI determination. We also agree with the pole value for the E2/M1 ratio found by the Mainz group. Our determinations confirm the view that this ratio is relatively model-independent. Given our agreement with the Mainz pole value, our slightly lower value at the resonance point remains to be explained.

There are two tests which might help to clarify the discrepancy. First, the pole value found in Ref. [12] could be extrapolated to the resonance point [13]. Second, the multipoles which will come from a Mainz fit to $\gamma p \rightarrow p \pi^{0}$ and $\gamma p \rightarrow n \pi^{+}$data (used to determine E2/M1 at the resonance point [11]) could be 'speed plotted' to determine a pole value for this ratio. This would give three independent sets of both pole and resonance values. If the extrapolation to the pole is indeed model-independent (as it appears to be), it would be interesting to see whether amplitudes giving an E2/M1 ratio near $-2.5 \%$ (at the resonance point) extrapolate to a pole value close to that found here and in Ref. [12]. 


\section{ACKNOWLEDGMENTS}

We thank A. L'vov, R. Beck, and L. Tiator for helpful communications. This work was supported in part by a U.S. Department of Energy Grant DE-FG05-88ER40454. 


\section{REFERENCES}

[1] R.A. Arndt, I.I. Strakovsky, and R.L. Workman, Phys. Rev. C 53, 430 (1996).

[2] M. MacCormick et al., Phys. Rev. C 53, 41 (1996).

[3] J. Peise et al., Phys. Lett. B 384, 37 (1996).

[4] R.L. Crawford and W.T. Morton, Nucl. Phys. B211, 1 (1983).

[5] R.M. Davidson, N.C. Mukhopadhyay, and R.S. Wittman, Phys. Rev. D 43, 71 (1991).

[6] These solutions can be viewed using SAID with a TELNET call to clsaid.phys.vt.edu with userid: said, or through our WWW site at http://clsaid.phys.vt.edu.

[7] R.L. Workman, R.A. Arndt, and Z. Li, Phys. Rev. C 46, 1546 (1992).

[8] R.M. Barnett et al., Phys. Rev. D 54, 1 (1996).

[9] M. Khandaker and A.M. Sandorfi, Phys. Rev. D 51, 3966 (1995).

[10] A.M. Sandorfi, private communications.

[11] R. Beck et al., Phys. Rev. Lett. 78, 606 (1997). There appears to be a typographical error in Eq.(4) of this paper. The signs of the $\mathrm{M}_{1+}$ and $\mathrm{M}_{1-}$ multipoles should be reversed.

[12] O. Hanstein, D. Drechsel, and L. Tiator, Phys. Lett. B385, 45 (1996).

[13] L. Tiator has claimed a value of $-2.4 \%$ for the E2/M1 ratio at the resonance energy, using the amplitudes of Ref. [12]. A comparison of the resonant and pole values from several sources was given during the N* meeting at INT, Seattle, 1996 (World Scientific, to be published), edited by T.-S.H. Lee and W. Roberts. 
Table I. Comparison of recent determinations of the $\Delta(1232)$ decay amplitudes. Values have the units $10^{-3} \mathrm{GeV}^{-1 / 2}$. See the text for a discussion of the range of values quoted for the VPI analyses.

\begin{tabular}{ccc}
\hline Source & $\mathrm{A}_{1 / 2}$ & $\mathrm{~A}_{3 / 2}$ \\
\hline VPI (old) [1] & $-141 \pm 5$ & $-261 \pm 5$ \\
Glasgow [4] & $-145 \pm 15$ & $-263 \pm 26$ \\
RPI [5] & $-135 \pm 16$ & $-251 \pm 33$ \\
VPI & $-135 \pm 5$ & $-250 \pm 8$ \\
\hline
\end{tabular}


Table II. Comparison of recent values for the E2/M1 ratio evaluated at the pole $(\mathrm{P})$ and resonance $(\mathrm{R})$ positions. See the text for a discussion of the range of values quoted for the VPI analyses.

\begin{tabular}{ccc}
\hline Source & Location & Value \\
\hline RPI [5] & $\mathrm{R}$ & $-1.57 \pm 0.72 \%$ \\
VPI (old) [7] & $\mathrm{R}$ & $-1.5 \pm 0.5 \%$ \\
BNL [9] & $\mathrm{R}$ & $-2.7 \%$ \\
Mainz [1] & $\mathrm{R}$ & $-2.5 \pm 0.2 \pm 0.2 \%$ \\
VPI & $\mathrm{R}$ & $-1.5 \pm 0.5 \%$ \\
\hline Mainz [12 & $\mathrm{P}$ & $-0.035-\mathrm{i} 0.046$ \\
VPI & $\mathrm{P}$ & $-0.034 \pm 5-\mathrm{i} 0.055 \pm 5$ \\
\hline
\end{tabular}

